\title{
Validation Framework of the Finite Element Modeling of Liver Tissue
}

\author{
Hongjian Shi, Rachid Fahmi, and Aly A. Farag \\ Computer Vision and Image Processing Laboratory (CVIP), \\ University of Louisville, Louisville, KY 40292 \\ \{hshi, rachidf, farag\}@cvip.louisville.edu
}

\begin{abstract}
In this work, we aim at validating some soft tissue deformation models using high resolution Micro Computed Tomography (Micro$\mathrm{CT}$ ) and medium resolution Cone-Beam CT (CBCT) images. These imaging techniques play a key role in detecting the tissue deformation details in the contact region between the tissue and the surgical tool (probe) even for small force loads, and provide good capabilities for creating accurate 3D models of tissues. Surgical simulations rely on accurate representation of the mechanical response of soft tissues subjected to surgical manipulations. Several finite element (F.E.) models have been suggested to characterize soft tissues. However, validating these models for specific tissues still remains a challenge. For our validation, ex vivo lamb liver is chosen to validate the linear elastic model (LEM), the linear viscoelastic model (LVEM), and the neo-Hooke hyperelastic model (NHM). We found that the LEM is more applicable to lamb liver than the LVEM for small force loads $(<40 \mathrm{~g})$ and that the NHM is closer to reality than the LVEM for this same range of force loads.
\end{abstract}

\section{Introduction}

Computer-aided surgical simulation progressed significantly in the last decade [1. 3]. Careful planning is of great importance in order to limit the damage to healthy tissue during surgery. In particular, accurate modeling of the mechanical behavior of the tissues is required. To achieve accurate prediction, biomechanical F.E. modeling of soft tissues is employed. The simplest one of such models is the LEM which was used early to estimate the force driven left ventricular deformation (e.g., [1]). Because of its simplicity, the LEM was widely used in early surgical simulation and is still used frequently [2, 4]. With the increasing speed of computer processing, the prediction accuracy attracts more attention. More complicated biomechanical models have been then employed to predict the deformations or registrations of brain, liver, skin and muscle tissues [4,5]. Some works on the liver tissue modeling using the FEM were reviewed and reported in 3 . However, the validation of these models for the liver tissue is still a challenging step in building a real surgical simulator for clinical use.

In this paper, we propose a validation framework and use it to validate the LEM, the LVEM, and the NHM of liver tissue. The tissue's mechanical properties 
are acquired by material testing using a Dynamic Materials Analyzer (DMA). A chamber consisting of a cylindrical plexiglass compartment, and a piece of liver are used for Micro-CT and CBCT scanning (details of this design can be found in [6]). These types of imaging can produce high resolution images. As a benefit, the deformation details in the contact region between the tissue and the probe can be captured and rendered even at small force loads ranging from several grams to tens of grams. Two techniques are used to validate these models. Firstly, we measure and compare the volume difference of the deformed object from simulations and the deformed object from experiments, as well as the vertical displacements of the probe from the experiments and the simulations. Secondly, the displacements of each vertex of the F.E. mesh are acquired and their root mean squares (RMS) for the three models, are computed and compared.

\section{Finite Element Models of Soft Tissues}

In this section, we will simply introduce the general framework of the LEM, the LVEM, and the NHM.

\subsection{Linear Elastic Model}

For the LEM, the material is assumed homogeneous and isotropic: the stress $\sigma$ and strain $\epsilon$ are directly proportional to each other via the Hooke's law $\sigma=\mathbf{D} \epsilon$. The matrix $D$ describes the material characteristics and depends on the Young's modulus and the Poisson's ratio (e.g., 7, 8, ). The relation between the stress and the displacement $\mathbf{u}$ can be written as $\epsilon=\mathbf{B u}$. Linear tetrahedral elements are used in our FE simulation. We denote by $\mathbf{u}_{i}^{e}$ the four nodal displacements of such elements. These displacements are used to compute the displacement inside each element as a linear combination of the shape functions $N_{i}^{e}(\cdot)$. Figure 1 shows the domain subdivision into finite elements and a linear tetrahedral element. For
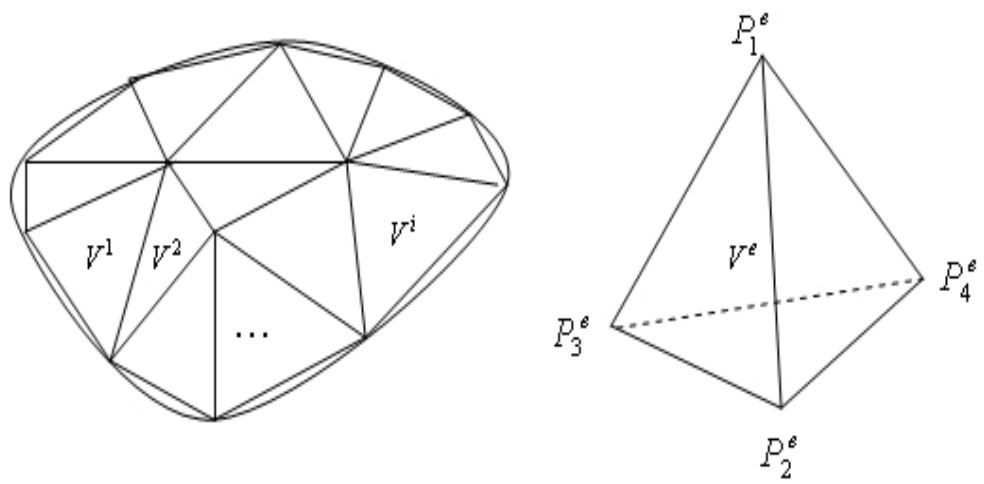

Fig. 1. Left: Subdivision of the domain into finite elements. Right: linear tetrahedral finite element. $V^{e}$ : element; $P_{i}$ 's: nodes. 
each element $V^{e}$, the potential energy in terms of the total element compound displacement vector $\mathbf{u}^{e}=\left[u_{1}^{e^{T}}, u_{2}^{e^{T}}, u_{3}^{e^{T}}, u_{4}^{e^{T}}\right]$ is:

$$
E^{e}\left(\mathbf{u}^{e}\right)=\frac{1}{2} \int_{V^{e}}\left(\mathbf{u}^{e}\right)^{T} \mathbf{B}^{e^{T}} \mathbf{D} \mathbf{B}^{e} \mathbf{u}^{e} d V^{e}-\int_{V^{e}} \mathbf{f}^{e^{T}} \mathbf{u}^{e} d V^{e}
$$

where $\mathbf{f}^{e}$ is the external force acting on the element $V^{e}$, and $\mathbf{B}^{e}$ is a constant matrix depending on the coefficients of the polynomial shape functions $N_{i}^{e}(i=$ $1,2,3,4)$ 9. The global equilibrium equation, obtained by minimizing the total potential energy $\left(\sum_{e} E^{e}\right)$, can be derived as a linear equation: $K U=F$. The dimension of the global stiffness matrix $K$ is $3 m \times 3 m$ and the dimension of the global force vector $F$ is $3 m$, where $m$ is the total number of nodes.

\subsection{Linear Viscoelastic Model}

A viscoelastic material shows a combination of viscous and elastic effects. The response of a viscoelastic material depends on both the current stress and the stress history up to the current time. Some soft tissues, such as liver, exhibit the viscoelastic property. One simple material model is the direct sum of the linear elastic effect and the linear viscous effect: $\epsilon(t)=\epsilon_{e}(t)+\epsilon_{v}(t)$ where $\epsilon_{e}(t)$ and $\epsilon_{v}(t)$ represent the strains in the elastic deformation and viscous flow respectively. Note that $\sigma(t)=E \epsilon_{e}(t)$, and $\sigma(t)=\eta \dot{\epsilon}_{v}(t)$ where $\eta>0$ is the Newtonian viscosity and $E$ is the Young's modulus. One can derive the constitutive equation of this model as follows (e.g., [7, 10]):

$$
\dot{\epsilon}(t)=\frac{\dot{\sigma}(t)}{E}+\frac{\sigma(t)}{\eta} .
$$

In our simulation, the F.E. software Abaqus [11] will use the stress relaxation test data for the viscoelastic modeling.

\subsection{Hyperelastic Model}

Hyperelastic materials are described in terms of a strain energy potential. There are several forms of strain energy potentials available in Abaqus FEM software 11 to model hyperelastic materials. One of them is the neo-Hooke's modeling. The material is assumed isotropic, and the energy potential function $W$ is approximated as follows:

$$
W=C_{10}\left(\bar{I}_{1}-3\right)+\frac{1}{D_{1}}\left(J^{e l}-1\right)^{2},
$$

where $C_{10}$ and $D_{1}$ are material parameters; $\bar{I}_{1}$ is the first deviatoric strain invariant defined as $\overline{I_{1}}={\overline{\lambda_{1}}}^{2}+{\overline{\lambda_{2}}}^{2}+{\overline{\lambda_{3}}}^{2}$, where the deviatoric stretches $\overline{\lambda_{i}}=J^{-1 / 3} \lambda_{i}$; $J$ is the total volume ratio; $J^{e l}$ is the elastic volume ratio; and $\lambda_{i}$ are the principal stretches. The initial shear modulus and bulk modulus are given by $\mu_{0}=2 C_{10}$ and $K_{0}=2 / D_{1}$. More details about the NHM and other hyperelastic models can be found elsewhere (e.g., [9, 11]). 


\section{Preprocessing of Soft Tissues}

To perform F.E. analysis of soft tissue deformation, preprocessing is needed: image segmentation, mesh generation, and estimation of the mechanical properties of the tissue.

Mesh generation techniques have been developed for different contexts and were aimed at different applications. In this work, we are interested in generating
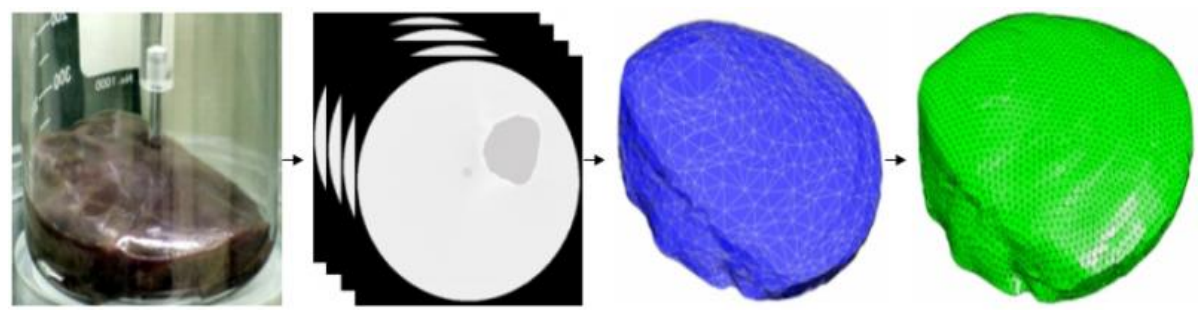

Fig. 2. From left to right, image acquisition, segmentation, stl conversion, and volume mesh generation
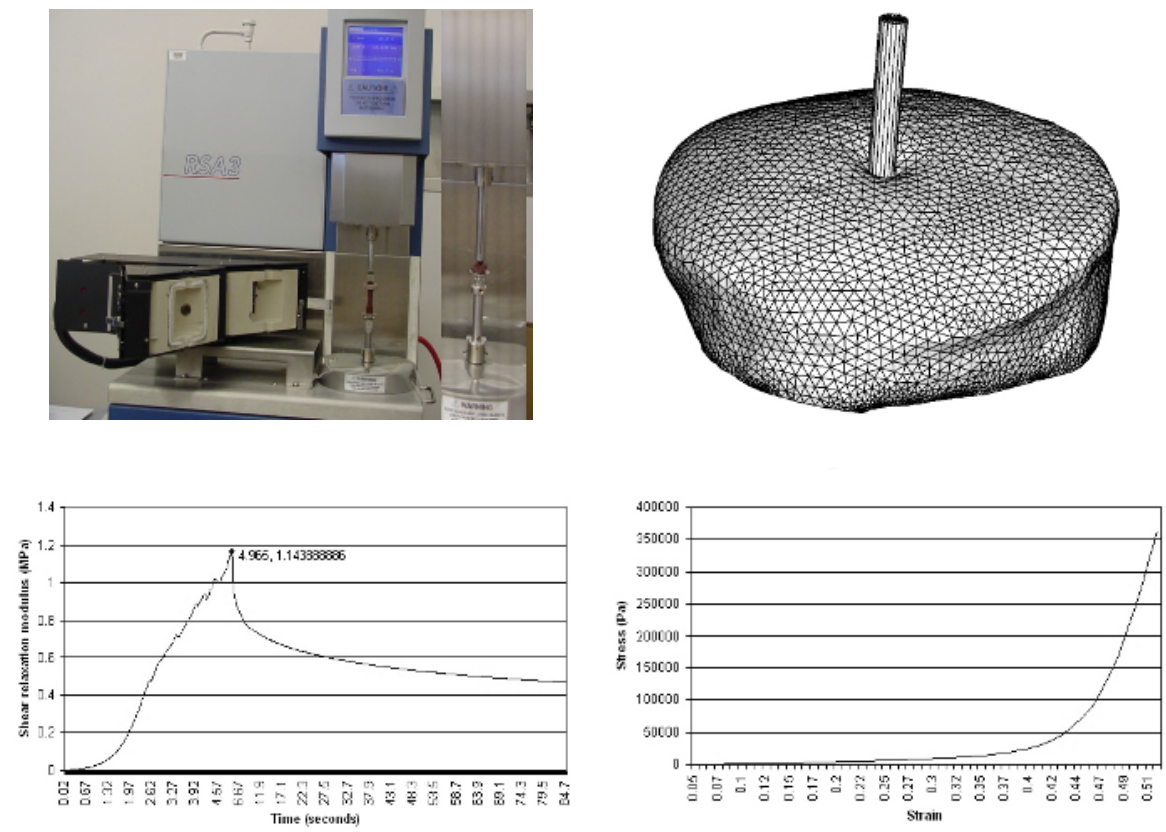

Fig. 3. Left to right: the DMA machine and deformed tissue with probe (top); the shear relaxation modulus curve; the stress vs. strain curve for strain less than $10.5 \%$ (zoomed) (bottom) 
high quality tetrahedral meshes that conform to the input surface meshes. For this purpose, a level set based segmentation approach [12] is employed. Furthermore, the segmented images are converted to a stereolithographic file which is input into NetGen [13] to generate a 3D mesh of the liver. The NetGen uses the advancing front technique combined with Delaunay tessellation. Figure 2 shows our procedure to generate volume meshes from a stack of medical images.

A second step of the preprocessing phase consists in measuring the mechanical properties of the liver tissue. Generally, these properties can be measured either in vivo [14, 16] or ex vivo [17. In this work, we cut two pieces of lamb liver, one with dimensions of $0.5 \times 2 \times 0.15$ in that we use for an extension test. The other piece, having a cylindrical shape with height of $0.5 \mathrm{in}$, is used for a compression test. These tests are performed using the DMA machine (Fig. 3. top-left). The shear relaxation test data (Fig. 3. bottom-left) are used by Abaqus software for the viscoelastic modeling. From the compression test, we got the global stress-strain curve (Fig. 3. bottom-right). The portion of the stress-strain curve for the strain less than $10.5 \%$ is used to compute the Young's modulus yielding $E=11055 P a$. The Poisson's ratio of 0.4 is used from literature [16, 17]. For the NHM, the initial shear modulus $\mu_{0}=3948 \mathrm{~Pa}$ and bulk modulus $K_{0}=18425$ $\mathrm{Pa}$ are computed from the stress-strain curve for strain less than $10.5 \%$.

\section{Proposed Finite Element Model Validation System}

In this section, we present a validation standard of F.E. models to verify the LEM, and the LVEM, and the NHM.

Experimental Setup: We developed a general purpose device ("testing chamber") that can be used to induce measurable deformations, which can be captured by two imaging sources: high resolution Micro-CT or medium resolution CBCT. The testing chamber is formed of a cylindrical plexiglass compartment. The tissue is placed on the bottom of the compartment and an aluminium probe with flat tip touches the upper surface of the tissue. Forces are applied by adding weights to a lever causing the probe to deform the tissue. The proposed soft tissue validation procedure can be summarized as follows. We first scan a piece of soft tissue using Micro-CT or CBCT machine to collect a stack of images of the undeformed tissue. Four force loads (5, 10, 20 and 40 grams) are gradually applied to the lever of the chamber. For each force load, we scan the deformed tissue when the equilibrium is reached. The collected images, both for the undeformed tissue and the deformed tissues, are then segmented and volume meshes are generated. We select a F.E. model (LEM, LVEM, or NHM) to perform F.E. analysis on the meshed undeformed object with the same force loads and same boundary conditions as those for the object in the experiment (see Fig. 3.topright for deformed object). Finally, we superimpose the deformed objects from simulation and the corresponding ones in the experiment, and we compute their volume difference and measure the vertical displacements of the probe tip in the experiment and the simulation for comparison purpose. 


\section{$5 \quad$ Validation Results}

To compute the deformation volume difference between the deformed object from simulation and the one from experiment, and its percentage with respect to the real deformation volume, we use a cylinder with radius of $20 \mathrm{~mm}$ and height of $20 \mathrm{~mm}$ to cover the deformed region. The cylinder is positioned vertically and centered at the probe contact and the top surface of the cylinder takes the shape of the undeformed liver surface. The deformed volume is computed by the number of voxels between the deformed surface and the top surface of the cylinder. The vertical displacements of the probe tip in real deformation and simulation are estimated from the acquired images and from the simulated object. For the LEM, the LVEM, and the NHM, all related volume differences, volume difference ratios, and the vertical displacements of the probe tip are listed in Table 11 where the abbreviations RD, LED, LVED and NHD stand for the vertical displacements of the probe tip in real deformation, and in simulation using the LEM, the LVEM and the NHD, respectively. RDV, LEVD, LVEVD, NHVD, LEVDP, LVEVDP, and NHVDP stand for the real deformation volume, the volume differences using the LEM, the LVM, and the NHM and their volume difference percentages, respectively. Figure 4 shows the superimposing of the deformed liver from simulation and the real deformed liver from experiments. From Table 1 and Fig. 4, we see that for small force loads $(<40 \mathrm{~g})$, the LEM is more applicable to the modeling of the lamb liver tissue. One can conclude that the liver deformation using the LEM is gradually going deep with the increasing force load. At the force load of 40 grams, the liver deformation using the LEM is larger than the real deformation. The liver deformations using the LVEM and the NHM are gradually approaching the real deformation with the increasing force load but the later is approaching faster. The root mean square values of the nodal displacements for each F.E. model are computed for different force loads (Table 2). These results are consistent with the results in Table 1 The strain

Table 1. Vertical displacements of the probe tip, volume differences and their percentages with respect to the real deformed volumes

\begin{tabular}{|c|c|c|c|c|c|c|c|c|c|c|c|}
\hline & RD & LED & LVED & NHD & RDV & LEVD & LEVDP & LVEVD & LVEVDP & NHVD & NHVDP \\
& $m m$ & $m m$ & $m m$ & $m m$ & $m^{3}$ & $m^{3}$ & $\%$ & $m^{3}$ & $\%$ & $m^{3}$ & $\%$ \\
\hline $5 \mathrm{~g}$ & 1.8 & 0.77 & 0.38 & 0.68 & 956 & 191 & 19.97 & 228 & 23.85 & 220 & 23.01 \\
$10 \mathrm{~g}$ & 2.6 & 1.54 & 1.18 & 1.32 & 1075 & 240 & 22.33 & 316 & 29.40 & 302 & 28.01 \\
$20 \mathrm{~g}$ & 4.2 & 4.39 & 2.36 & 2.57 & 1241 & 245 & 19.74 & 382 & 30.78 & 359 & 28.93 \\
$40 \mathrm{~g}$ & 6.2 & 9.59 & 4.71 & 5.04 & 1527 & 299 & 19.58 & 506 & 33.14 & 476 & 31.17 \\
\hline
\end{tabular}

Table 2. RMS values (mm) for each F.E. model for different force loads

\begin{tabular}{|c|c|c|c|c|}
\hline & $5 \mathrm{~g}$ & $10 \mathrm{~g}$ & $20 \mathrm{~g}$ & $40 \mathrm{~g}$ \\
\hline LEM & 0.0487956 & 0.097591 & 0.195182 & 0.394411 \\
LVEM & 0.025235 & 0.050470 & 0.100939 & 0.201876 \\
NHM & 0.030717 & 0.061107 & 0.121318 & 0.239742 \\
\hline
\end{tabular}



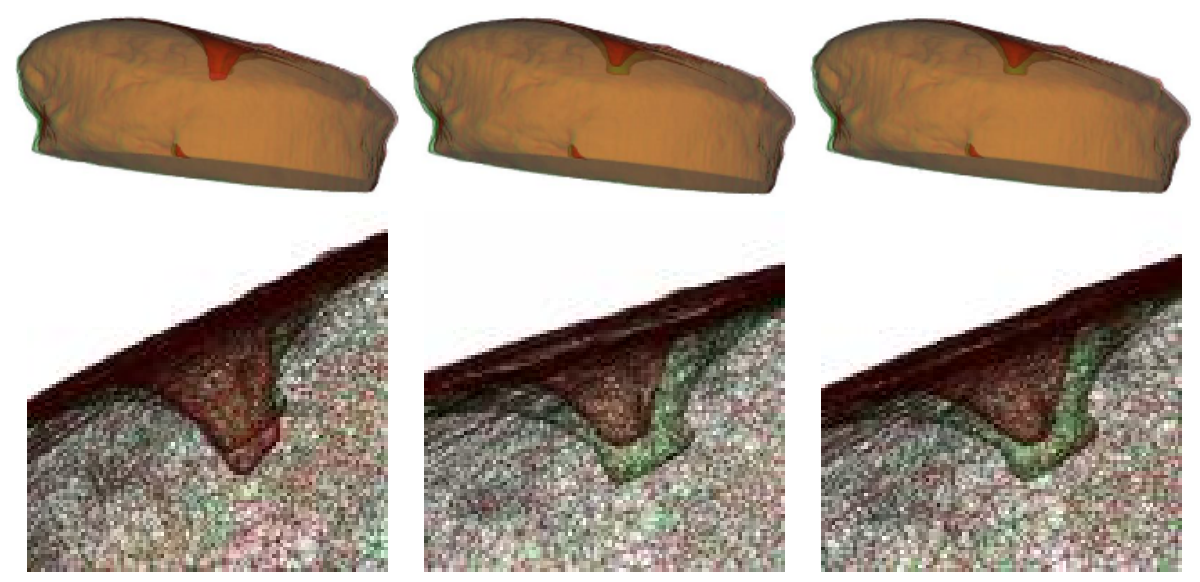

Fig. 4. Left to right: superimposing of the simulated livers and the real deformed ones under the force load 40 grams for the LEM, the LVEM, and the NHM, respectively. Wireframed zooms around the contact region are shown in the second row.

repartition plots from Abaqus for the three F.E. models and for force loads up to 40 grams, show that the maximal strains do not exceed $10 \%$. This confirms that all simulations are carried out in the small deformation framework. The viscous component only governs the time dependent behavior of the tissue and the final displacement for any given load will only depend on the elastic property. The observed difference in displacements for the LEM and the LVEM is related to the fact that the LVEM accounted for time behavior or the reported displacements were not at the time of infinity, the elastic components of the two models might be different. Also, it should be pointed out that there is a geometric nonlinearity in the experiment and the simulation. As larger force is applied to the probe, it pushes into more tissue. Consequently, there is more surface area in contact with the probe to provide support. For the NHM, the geometric nonlinearity is considered in simulation.

\section{Conclusion}

We presented F.E. modeling of soft tissues from Micro-CT or CBCT images. These types of images show the volume and surface shape changes for small force loads. The F.E. method was used to analyze the lamb liver deformations under certain force loads using the LEM, the LVEM, and the NHM. The deformations were measured from the models and compared to the real deformations measured by the experiment setup. We found that the LEM is more applicable to lamb liver than the LVEM for small force loads $(<40 \mathrm{~g})$ and that the NHM is closer to reality than the LVEM for this same range of force loads. The comparability of the results presented herein to the in vivo tissue, which is valuable 
for future surgery simulation design, is beyond the scope of this work and will be investigated in future studies.

\section{References}

1. Shi, P., Robinson, G., Constable, R.T., Sinusas, A., Duncan, J.: A model-based integrated approach to track myocardial deformation using displacement and velocity constraints. In: Fifth International Conference on Computer Vision. (1995) 687-692

2. Bro-Nielsen, M.: Finite element modeling in surgery simulation. Proceedings of the IEEE 83 (1998) 490-503

3. Delingette, H., Ayache, N.: Soft tissue modeling for surgery simulation. In Ayache, N., ed.: Computational Models for the Human Body. Handbook of Numerical Analysis (Ed : Ph. Ciarlet). Elsevier (2004) 453-550

4. Skrinjar, O.M., Spencer, D., Duncan, J.S.: Brain shift modeling for use in neurosurgery. In: MICCAI '98: Proceedings of the First International Conference on Medical Image Computing and Computer-Assisted Intervention, London, UK, Springer-Verlag (1998) 641-649

5. Larrabee, W.: A finite element model of skin deformation: biomechanics of skin and tissue: a review. Laryngoscope 96 (1986) 399-405

6. Farag, A., Shi, H., Fahmi R., Voor, M.: Basic experiments and finite element analysis of soft tissues. In Payan, Y., ed.: Biomechanics Applied to Computer Assisted Surgery. Research Signpost (2005)

7. Fung, Y.C.: Biomechanics: Mechanical Properties of Living Tissues. SpringerVerlag, New York (1981)

8. Reddy, J.N.: Energy and Variatiobal Methods in Applied Mechanics. John \& Sons, Inc (1984)

9. Zienkiewicz, O.C., Taylor, R.L.: The Finite Element Method. Fifth edn. Butterworth-Heinemann (2000)

10. Creus, G.J.: Viscoelasticity - basic theory and applications to concrete structures. In Brebbia, C.A., Orszag, S.A., eds.: Lecture Notes in Engineering. Volume 17. Springer-Verlag (1986)

11. Abaqus: Abaqus User's Manuals, Abaqus Version 6.4. HKS Inc. (2003)

12. Farag, A.A., Hassan, H.S.: Adaptive segmentation of multi-modal 3d data using robust level set techniques. In: MICCAI (1). (2004) 143-150

13. Schöberl, J.: Netgen - an advancing front $2 \mathrm{~d} / 3 \mathrm{~d}$-mesh generator based on abstract rules. Comput. Visual. Sci. (1) 41-52

14. Carter, F.J., Frank, T.G., Davies, P.J., McLean, D., Cuschieri, A.: Measurements and modeling of the compliance of human and porcine organs. Medical Image Analysis 5 (2001) 231-236

15. Brouwer, I., Ustin, J., Bentley, L., Sherman, A., Dhruv, N., Tendick, F.: Measuring in vivo animal soft tissue properties for haptic modeling in surgical simulation. In: Medicine Meets Virtual Reality 2001. (2001) 69-74

16. Ottensmeyer, M.P., Kerdok, A.E., Howe, R.D., Dawson, S.: The effects of testing environment on the viscoelastic properties of soft tissues. In: ISMS. (2004) 9-18

17. Hu, T., Desai, J.P.: A biomechanical model of the liver for reality-based haptic feedback. In: MICCAI (1). (2003) 75-82 